\title{
Adaptation to displaced vision: Evidence for transfer of adaptation and long-lasting aftereffects
}

\author{
BETSY R. YACHZEL and JAMES R. LACKNER \\ Brandeis University, Waltham, Massachusetts 02154
}

\begin{abstract}
Aftereffects following a single exposure to visual rearrangement last for $48 \mathrm{~h}$ and longer. Following multiple spaced exposures to visual rearrangement, aftereffects persisted for at least 2 weeks. Over the course of the multiple exposures, subjects showed diminished scatter when pointing without sight of hand to visual targets but did not show diminished constant errors. Adaptation achieved in one visuomotor transferred nearly perfectly to a test situation involving somewhat different movement demands.
\end{abstract}

The human sensory-motor system responds to external perturbations in a plastic and malleable fashion, exhibiting homeostatic changes that compensate for distorting, illusory, or stressful sensory stimulation. Ebenholtz $(1966,1973)$ has shown that adaptation to tilt of the visual field is dependent on the rate of introduction of tilt; too rapid increments in tilt may actually inhibit adaptation. Lackner and Lobovits (1977, Note 1) recently investigated how different ways of introducing lateral visual displacement influenced the amount of homeostatic compensation that would be achieved; they concluded that vestibular adaptation and visuomotor adaptation might share certain dynamic characteristics. Graybiel earlier had shown that adaptation to the stressful cross-coupled angular accelerations generated when head movements are made in a slow rotation room was facilitated if the room was brought up to terminal velocity incrementally rather than in a single step (Graybiel, Deane, Colehour, Note 2; Graybiel \& Wood, Note 3). Graybiel and Knepton (1972) also established that long-term (nondirection-specific) as well as short-term (direction-specific) vestibular adaptation was enhanced by an incremental exposure schedule. Lackner and Lobovits (1977, Note 1) found an analogous facilitation of sensorymotor adaptation to visual rearrangement when comparing stepwise exposure conditions with singlestep exposure: as a function of average visual displacement over time, stepwise exposure produced greater adaptation. In addition, aftereffects persisted more than $24 \mathrm{~h}$ after the end of the exposure period,

This study was supported by grants from the Rosenstiel Biomedical Sciences Foundation and the Spencer Foundation. We thank Ronald Calvanio for statistical assistance. Reprint requests should be sent to James R. Lackner, Department of Psychology, Brandeis University, Waltham, Massachusetts 02154. James R. Lackner is also at the Department of Psychology, Massachusetts Institute of Technology, Cambridge, Massachusetts 02139. although no significant difference in aftereffect magnitude was seen between the single-step and stepwise exposure paradigms.

Such aftereffects represent a persistence of compensatory changes; subjects remain in the newly adapted state for some period after the adaptationinducing stimulation is ended. Despite these preliminary observations, little is known about how the presence of aftereffects influences adaptation during subsequent exposures to visual rearrangement. Graybiel's experiments in the slow rotation room have shown that vestibular adaptation to rotation achieved with an incremental exposure paradigm does not decay rapidly; in fact, with weekly practice, subjects not only retain adaptation, but improve on it, indicating that definite carry-over effects exist.

The present experiment was designed to see if similar principles govern the adaptation elicited during exposure to visual rearrangement. Subjects were given six identical conditions of exposure to visual displacement within a 2 -week period, and the effects of repeated exposure on the amounts of adaptation in each exposure session were examined as well as the persistence of aftereffects. Half of the subjects received stepwise exposure conditions and half received single-step exposure to determine whether stepwise exposure might increase aftereffect duration just as it facilitates adaptation. Since aftereffects have been shown to last as long as $24 \mathrm{~h}$, longer term persistence was measured using a 48 - $h$ delay from one exposure to the next and then again after a 2-week delay period following the last exposure condition.

Another question dealt with was whether adaptation transfers from one visuomotor environment to another or whether it is a form of context-dependent learning. Graybiel, Thompson, Deane, Fregley, Colehour, and Ricks (1968) found that vestibular adaptation achieved in one motion environment 
tends to transfer to other motion environments. In the slow rotation room, for example, transfer occurs if the subject is changed from a horizontal orientation to a vertical one, or vice versa; accordingly, vestibular adaptation is not restricted only to the specific stimulation contexts in which it is achieved. To determine whether the same would be true of adaptation to visual rearrangement, transfer of adaptation was measured by exposing subjects to visual rearrangement in one visuomotor environment and then testing them in another visuomotor environment involving different kinds of movements. It was of special interest to compare the influence of stepwise and single-step exposure conditions on transfer of adaptation, because stepwise exposure facilitates the transfer of vestibular adaptation.

The experimental paradigm provided as a sidebenefit the possibility of determining whether accuracy and consistency in pointing at visual targets improves from exposure session to exposure session even though the subject sees his hand only during the exposure period and never when pointing to the visual targets.

\section{METHOD}

\section{Subjects}

Twelve right-handed Brandeis students, four males and eight females, participated voluntarily; their ages ranged from 18 to 22 years. None of them had visual or motor impairments that might have degraded their performance.

\section{Experimental Procedure}

The experiment took place over a 5-week period and was divided into three stages. The first stage involved two practice preexposure sessions in a Held-Gottlieb (1958) type of device on different days within the same week. These sessions were included before the experimental ones to familiarize the subjects with the demands of the visuomotor coordination task and to provide them with practice in pointing without sight of the hand. On both days, the preexposure trials were identical: The subject, with his head stabilized by a biteplate, pointed in succession to four visual targets. The targets were reflections of the heads of white stick pins that had been set in a corkboard surface to form the corners of a $15.24 \times 15.24 \mathrm{~cm}$ square; a first surface mirror reflected the targets into the subject's field of view so that they appeared $62.7 \mathrm{~cm}$ in front of him, symmetric to his sagittal plane, and below the plane of the mirror; the mirror also prevented sight of the hand. When pointing at the visual targets, the subject held a pen-shaped stylus in his right hand. The stylus, on reaching the apparent plane of the visual targets, made contact with an electrically graded surface, thereby producing a reading on a digital voltmeter indicating the horizontal position of contact. The subject first pointed to the upper left-hand target and then continued in a clockwise direction around the square for a total of 20 cycles, resting his hand after each pointing movement, in order to make each response independent from the previous one, and proceeding at a comfortable speed while yet being as accurate as possible.

In the second stage, the subjects were required to come in six times (Sessions A-F), on Monday, Wednesday, and Friday for
2 consecutive weeks. On Day A (Monday), the subject first had a preexposure condition on a new machine different from the one used in the first stage. On the new machine (cf. Lackner, 1973), pointing responses to four visual targets were also measured, but the targets were metal pins located on the arc of a circle whose center was the midpoint of the subject's interocular axis. The targets were positioned $30^{\circ}$ left, $10^{\circ}$ left, $10^{\circ}$ right, and $30^{\circ}$ right in relation to the midline. The subject pointed with a pen-shaped stylus to a conductive surface just below the visual location of the target. Each time the stylus made contact, a voltage reading was recorded on a digital voltmeter, indicating the horizontal position of contact. Sight of the hand was prevented by an opaque horizontal shelf that extended forward at chin level. The subject pointed with his outstretched arm left to right for a total of 80 responses, resting his arm after each niark and proceeding at a comfortable speed. The averages of the 20 responses to each individual target were used as a measure of preexposure accuracy and as a reference for determining transfer of adaptation. After a rest period, the subject was transferred to the Held-Gottlieb apparatus where he proceeded through a preexposure trial as described earlier. The average of the 20 responses to each individual target was used as a reference to measure adaptation on each day and also as a baseline to compare later preexposure scores on subsequent days.

For the exposure period, the mirror was raised out of view and a panel was lowered to occupy the same plane as had the reflected visual array in the preexposure period. The panel surface held a $16.5 \times 16.5 \mathrm{~cm}$ square pattern formed by $1.27-\mathrm{cm}$ wide white tape. In the exposure period, the subject was allowed sight of his hand through prism spectacles. The subjects had been divided into two major groups: six received a single-step visual displacement, three getting a rightward and three a leftward displacement; the other six subjects received stepwise displacement, three getting a leftward and three a rightward displacement. All 12 subjects received a full $10 \mathrm{~min}$ of visual displacement: in single-step exposure, a 20-diopter visual displacement was present for the $10 \mathrm{~min}$; in stepwise exposure, the visual displacement was introduced incrementally-by means of a mechanical linkage system attached to binocular Risley prisms1 diopter every $30 \mathrm{sec}$, thereby achieving a 20-diopter displacement in the last $30 \mathrm{sec}$. During the exposure period, the subject traced the sides of the white square with his right index finger, starting at the upper left-hand corner and following the beat of a metronome ( 60 beats $/ \mathrm{min}$ ). The subject maintained an 8 beat count: during the first 4 beats, he traced the white square, and during the second 4 beats, he rested his hand.

After the exposure period, while the subject kept his eyes closed, the apparatus was returned to the preexposure configuration, a process that took approximately $15 \mathrm{sec}$. Then, for the postexposure period, the subject repeated the procedures of the preexposure period. The average of responses to each target was calculated and compared to the preexposure values, with the difference between post- and preexposure scores serving as a measure of adaptation.

The subject then came back on Wednesday ( $48 \mathrm{~h}$ later) and Friday of the same week and Monday, Wednesday, and Friday of the next week and repeated the procedures in the Held-Gottlieb apparatus on each of these days. On Friday of the second week after the postexposure period had been completed (i.e., after Session F), the subject with his eyes closed was led to the other machine and then tested again as earlier described. The average of the 20 pointing movements for each target was calculated and compared with his earlier scores in this device to provide a measure of transfer of adaptation.

Finally, each subject came back 2 weeks after Session $F$ and repeated the preexposure condition in the Held-Gottlieb apparatus. This performance was compared with his original preexposure scores obtained in Session $A$ to determine the presence of any long-term aftereffects. 


\section{RESULTS}

Responses were pooled across subjects within subconditions and averaged separately for each of the six exposure sessions as well as for the transfer of adaptation conditions and the long-term aftereffect measure. Aftereffects were examined (a) by comparing preexposure scores from session to session and doing a trend analysis, and comparing the first and last exposure sessions, (b) by noting if adaptation occurred in all sessions, and (c) by comparing the amount of adaptation achieved in a given session with the magnitude of the aftereffect persisting from the prior exposure condition.

A one-way analysis of variance showed that the preexposure means from Sessions A-F, collapsed across exposure type and direction of visual displacement, were significantly different $(p<.05)$. A trend analysis indicated that there were significant differences between preexposure scores on Session A and all subsequent sessions except $B(p<.05)$ and between preexposure scores on Session $B$ and all subsequent sessions $(\mathrm{p}<.05)$; the remaining comparisons were nonsignificant. Additional analyses showed that a significant shift occurred in preexposure means from Session A to Session F, for both single-step and stepwise exposure conditions, as well as from Session $\mathrm{A}$ to Session $\mathrm{B}$, for the singlestep but not the stepwise conditions (Table 1). The preexposure mean shift was significant $(p<.01)$ when single-step and stepwise exposure conditions were collapsed. The shifts in preexposure mean scores demonstrate that aftereffects persist for at least $48 \mathrm{~h}$ after exposure to visual rearrangement.

Table 2 indicates that significant adaptation was acquired in four of the six single-step exposure sessions and five of the six stepwise exposure sessions. For both single-step and stepwise exposure conditions, there is a tendency for less adaptation to be achieved after Sessions A and B, although an analysis of variance showed this trend to be nonsignificant $(\mathrm{p}>.05)$.

Table 3 shows the persistence of long-term aftereffects 2 weeks after the last exposure conditions; the aftereffect was calculated by subtracting the preexposure means on Session A from the scores obtained 2 weeks after the last exposure condition. When the adaptation achieved in Session $A$ is compared across exposure types with the aftereffect that was measured 2 weeks after the last exposure period, the initial adaptation, $3.18^{\circ}$, is significantly larger than the persisting aftereffect, $.97^{\circ}$, indicating a partial decay of adaptation $(p<.025)$. Table 4 indicates that transfer of adaptation occurred from. one sensory-motor environment to another both for the single-step and the stepwise exposure conditions.

A comparison of single-step and stepwise exposure conditions for differences in preexposure mean
Table 1

Mean Preexposure Shifts in Degrees for Single-Step and Stepwise Exposure Conditions Between Sessions $A$ and $F$ and Between Sessions $A$ and $B$

\begin{tabular}{lllll} 
& & & \multicolumn{2}{c}{ Preexposure Shift } \\
\cline { 5 - 5 } Condition & Sessions & & $\mathrm{M}$ & $\mathrm{SD}$ \\
\hline Single-Step & F-A & & $1.33^{*}$ & 1.50 \\
Stepwise & F-A & & $1.67^{* *}$ & 1.36 \\
Single-Step & B-A & & $2.00^{* *}$ & 1.67 \\
Stepwise & B-A & .67 & 1.36 \\
\hline
\end{tabular}

Note-Each entry represents the average of six subjects. *Significantly different from zero by one-tailed t test $(p<.05)$. $* *(p<.025)$

Table 2

Mean Adaptation in Degrees for Single-Step and Stepwise Exposure Condition: Sessions $A$ to $F$

\begin{tabular}{clllll} 
& \multicolumn{2}{c}{ Single-Step } & & \multicolumn{2}{c}{ Stepwise } \\
\cline { 6 - 6 } \cline { 5 - 6 } Session & \multicolumn{1}{c}{$\mathbf{M}$} & SD & & \multicolumn{1}{c}{ M } & SD \\
\hline A & $4.35^{* *}$ & 3.06 & & 2.02 & 2.57 \\
B & $2.22^{* *}$ & 1.48 & & $2.61 \dagger$ & 1.49 \\
C & $1.77^{*}$ & 1.88 & & $1.48^{*}$ & 1.51 \\
D & 1.17 & 1.71 & & $1.77^{* *}$ & 1.16 \\
E & 1.64 & 2.03 & & $1.94^{* *}$ & 1.26 \\
F & $1.25^{*}$ & 1.46 & & $1.83^{*}$ & .64 \\
\hline
\end{tabular}

Note-Each entry represents the average of six subjects. *Significantly different from zero by one-tailed t test $(p<.05)$. $* *(p<.01) \quad+(p<.005)$

Table 3

Magnitude of Long-Term Aftereffect in Degrees

\begin{tabular}{lcc} 
& \multicolumn{2}{c}{ Aftereffect } \\
\cline { 2 - 3 } \multicolumn{1}{c}{ Condition } & $\mathbf{M}$ & SD \\
\hline Single-Step and Stepwise & $.99^{*}$ & 1.13 \\
Single-Step & $1.16^{*}$ & 1.33 \\
Stepwise & .81 & 1.24 \\
\hline
\end{tabular}

Note-For the collapsed single-step and stepwise conditions, each entry represents the average of 12 subjects; for the single step and stepwise entries there are 6 subjects for each entry. Aftereffects were calculated by subtracting preexposure scores on Session $A$ from the scores obtained 2 weeks after the last exposure condition.

${ }^{*}$ Significantly different from zero by one-tailed t test $(p<.01)$

Table 4

Mean Transfer of Adaptation in Degrees for Single-Step and Stepwise Exposure Conditions

\begin{tabular}{ccc} 
& \multicolumn{2}{c}{ Transfer of Adaptation } \\
\cline { 2 - 3 } Condition & \multicolumn{1}{c}{$\mathbf{M}$} & SD \\
\hline Single-Step & $3.31^{* *}$ & 2.86 \\
Stepwise & $2.31^{*}$ & 2.37 \\
\hline
\end{tabular}

Note-Each entry represents the average of six subjects. *Significantly different from zero by one-tailed $t$ test $(p<.05)$. $* *(p<.025)$ 
scores between Sessions A and B and Sessions A and $F$ failed to reach significance. The differences in mean adaptation for single-step and stepwise exposure conditions were examined for all six exposure conditions; the results were not significant. Nor were the stepwise and single-step exposure conditions different in terms of magnitudes of long-term aftereffects and transfer of adaptation.

The influence of direction of visual rearrangement on magnitude of adaptation was also examined for the six exposure sessions; although no significant differences appeared, rightward visual displacements tended to elicit greater adaptation.

Plots were made of each subject's standard deviations and mean pointing accuracy for each of the four visual targets in preexposure sessions, A through F. An examination of these plots revealed increased accuracy from Session $A$ to Session $F$ in terms of smaller standard deviations, although there were no systematic trends from session to session (cf. Table 2). No decrease in constant errors was present.

\section{DISCUSSION}

It is evident that carryover effects from exposure to visual rearrangement persisted for $48 \mathrm{~h}$ and longer, as indicated by significant shifts in preexposure mean scores from session to session (A to $\mathrm{B}$ and $\mathrm{A}$ to $\mathrm{F}$ ) and by the significantly smaller amount of adaptation occurring in Session F than in Session A. The long-term aftereffects that were present 2 weeks after the last exposure condition are of considerable interest, because subjects reported no inaccuracies in visuomotor coordination when reaching for external objects during the 2 -week period following Session F; nevertheless, when pointing without visual feedback in the Held-Gottlieb apparatus, they showed significant errors. It seems likely that subjects did not show reaching errors in natural situations between testing sessions because they then had sight both of their hands and external targets and, consequently, continuous visual feedback about the relative positions of hand and target. Conscious correction on the part of the subjects seems unlikely because there is no reason they should not have continued such correction in the test apparatus, in which case the presence of the aftereffects would have been masked.

In related experiments, Redding $(1973,1975)$ has studied adaptation and decay of adaptation as a function of tilt and of displacement of the visual field. His results indicate that adaptation to visual displacement decays rapidly after a single exposure and is nearly absent in $56 \mathrm{~min}$; by contrast, adaptation to tilt is still present at measurable levels $56 \mathrm{~min}$ after the exposure period. This difference led Redding to suggest that adaptation to tilt involves a long-term component not shared by displacement adaptation. The findings of the present report indicate that a long-term component in adaptation to displacement can be demonstrated when subjects are given multiple exposures. Moreover, this longterm adaptation is not specific to the visuomotor environment in which adaptation was achieved because adaptation transferred, for both single-step and stepwise exposure conditions, to a test situation involving somewhat different hand and arm movements. In the Held-Gottlieb apparatus, the subject's arm motion involved movement at the shoulder, elbow, and wrist much like that in throwing darts; by contrast, in the other test apparatus, where the arm was held at a constant height with respect to the body and lateral shoulder movement, slight elbow and considerable wrist movement was involved. Such transfer indicates that, even though the aftereffects following adaptation to visual rearrangement are very long-lasting, these aftereffects are not specific to particular visuomotor acts performed during the exposure period. Accordingly, they have different properties than the long-lasting visual aftereffects, such as the McCullough effect (1965), which have recently been described. The latter aftereffect decreases in magnitude the more the specific characteristics of the test situation differ from the inducing situation (Skowbo, Timney, Gentry, \& Morant, 1975). Consequently, the question raised by Lackner and Lobovits (1977) as to whether or not sensorymotor aftereffects and visual aftereffects follow similar principles of manifestation must be answered in the negative.

No significant differences appeared between the single-step and stepwise exposure conditions in terms of facilitation of adaptation; nevertheless, all trends were in the appropriate direction despite the small number of subjects in each group, indicating that additional experimentation is warranted.

Subjects showed a decrease in scatter of responses for each of the four visual targets when Sessions A and F were compared. Lackner (1974) earlier had found that, in the absence of visual feedback, proprioceptive feedback enables subjects to become more accurate in pointing to visual targets. In the present situation, both visual and proprioceptive feedback were absent, yet subjects exhibited an overall improvement in accuracy. However, only a decrease in scatter of responses occurred, not a decrease in constant errors; by contrast, Lackner (1974) found that subjects permitted proprioceptive feedback about target location showed decreases in constant errors as well as in standard deviations. The persistence of undiminishing constant errors in the present experimental situation indicates that the subjects had no better idea of where the targets were in relation to themselves when the experiment was over than when it began. 


\section{REFERENCE NOTES}

1. Lackner, J. R., \& Lobovits, D. Incremental exposure facilitates adaptation to sensory rearrangement. Unpublished manuscript. 1976. (Available from J. R. Lackner, Department of Psychology, Brandeis University, Waltham, Massachusetts 02154.)

2. Graybiel, A., Deane, F. R., \& Colehour, J. K. Prevention of overt motion sickness by incremental exposure to otherwise highly stressful Coriolis accelerations. NASA-NAMI Joint Report, NAMI$1044,1968$.

3. Graybiel, A., \& Wood, C. D. Rapid vestibular adaptation in a rotating environment by means of controlled head movements. NASA-NAMI Joint Report, December 1968.

\section{REFERENCES}

Ebenholtz. S. M. Adaptation to a rotated visual field as a function of degree of tilt and exposure time. Journal of Experimental Psychology, 1966, 72, 629-634.

Ebenholtz, S. M. Optimal input rates for tilt adaptation. American Journal of Psychology, 1973, 86, 193-200.

Graybiel, A., \& KNEPTon, J. Direction-specific adaptation effects acquired in a slow rotation room. Aerospace Medicine, 1972, 43, 1179-1189.

Graybiel, A., Thompson, A. B., Deane, F. R., Fregly, A. R., ColehouR, J. K., \& Ricks, E. L., JR. Transfer of habituation of motion sickness on change in body position between vertical and horizontal in a rotating environment. Aerospace Medicine, $1968,39,950-962$.

Held, R., \& Gotrlieb, N. Technique for studying adaptation to disarranged hand-eye coordination. Perceptual and Motor Skills, 1958, 8, 83-86.

LACKNER, J. R. A device for investigating adaptation to visual rearrangement. Journal of Psychology, 1973, 85, 137-141.

LACKNER, J. R. Proprioceptive facilitation of open-loop visual pointing. Perceptual and Motor Skills, 1974, 39, 263-265.

LACKNER, J. R., \& LoBovirs, D. A. Adaptation to displaced vision: Evidence for prolonged aftereffects. Quarterly Journal of Experimental Psychology, 1977, 29, 65-69.

Mc Collough, C. Color adaptation of edge detectors in the human visual system. Science, 1965, 149, 1115-1116.

Redding, G. M. Visual adaptation to tilt and displacement: Same or different processes? Perception \& Psychophysics, $1973,14,193-200$

Redding, G. M. Decay of visual adaptation to tilt and displacement. Perception \& Psychophysics, 1975, 17, 203-208.

Skowbo, D., Timney, B. N., Gentry, T. A., \& Morant, R. B. McCollough effects: Experimental findings and theoretical accounts. Psychological Bulletin, 1975, 82, 497-510.

(Received for publication August 11, 1976; revision accepted May $7,1977$. 